\title{
Fumadores ocasionales y ligeros, prevalencia, importancia y alternativas de tratamiento
}

\section{Occasional and light smokers, prevalence, importance and treatment alternatives}

\author{
Andrea Hernández-Pérez,* Leonor García-Gómez,* Jennifer Osio-Echánove,* Rogelio Pérez-Padilla*
}

*Instituto Nacional de Enfermedades Respiratorias Ismael Cosío Villegas, Ciudad de México.

Palabras clave: Fumar, fumadores ocasionales, tabaquismo, fumadores ligeros.

Keywords: Smoking, occasional smokers, tobacco, light smokers.

En las estadísticas más recientes provenientes de las encuestas de salud (Encuesta Nacional de Consumo de Drogas, Alcohol y Tabaco $^{1}$ y Encuesta Global de Tabaquismo), ${ }^{2}$ se observa que la tasa de fumadores con un consumo diario se ha reducido a expensas de un crecimiento de quienes fuman ocasionalmente. En México, de los actuales 14.9 millones de individuos quienes fuman, 9.4 millones lo hacen de forma ocasional, siendo un $62 \%$, representando la mayor proporción. ${ }^{1}$ No es sólo una característica de México, sino que se presenta también en otros países. Por ejemplo, en Estados Unidos se reporta una prevalencia de $19.7 \%$ de fumadores esporádicos. ${ }^{3}$

Por ello resulta relevante explorar dicha población, considerando los patrones de utilización de tabaco, niveles de dependencia, características asociadas y alternativas de tratamiento específicas, atendiendo a las necesidades particulares identificadas.

\section{CARACTERÍSTICAS ASOCIADAS A LOS FUMADORES OCASIONALES}

De acuerdo a los indicadores establecidos por la Organización Mundial de la Salud, en particular del Sistema Global

Correspondencia:

Dr. Rogelio Pérez-Padilla

Instituto Nacional de Enfermedades Respiratorias Ismael Cosío

Villegas, Ciudad de México.

Correo electrónico: perezpad@gmail.com

Recibido: 03-XII-2020; aceptado: 07-XII-2020.

Citar como: Hernández-Pérez A, García-Gómez L, Osio-Echánove J, Pérez-Padilla R. Fumadores ocasionales y ligeros, prevalencia, importancia y alternativas de tratamiento. Neumol Cir Torax. 2021; 80 (2): 89-93. https:// dx.doi.org/10.35366/100988 de Vigilancia Epidemiológica del Tabaco, ${ }^{4}$ en el conjunto de preguntas acerca de esa planta fumable, se establece un patrón de empleo diario u ocasional partiendo de la frecuencia de consumo en el último mes; a quienes fuman todos los días al menos un cigarro se les considera «fumadores diarios» y aquellos que no lo hacen diariamente se consideran «fumadores ocasionales». Aunque en la literatura existen diferentes enfoques y medidas epidemiológicas para los denominados así, dicha inconsistencia se observa en la definición operacional dentro de este grupo de consumidores, ya que algunos autores definen las categorías a partir de la cantidad de cigarros consumidos por día, otros contemplan el número de días de uso, mientras que otros toman en cuenta el nivel de sumisión fisiológica.

Dentro de las características que se han descrito en la población de quienes fuman esporádicamente destacan: una baja dependencia a la nicotina, medida por el test de Fagerström, comparada con los que fuman cada día; menos probabilidades de tener depresión o ansiedad, o de consumir drogas ilícitas, numerosos intentos para dejar de fumar, si bien hay estudios los cuales refieren que el tipo de fumador (ligero, ocasional o diario) no predice el éxito en las tasas de abandono en los tratamientos de cesación: ${ }^{5-9}$

Características de fumadores ocasionales o intermitentes.

1. Menor percepción de riesgo en jóvenes. ${ }^{10}$

2. Edad de inicio más tardía en comparación con fumadores diarios. ${ }^{5}$

3. Identificarse como fumadores no activos. ${ }^{11}$

4. Baja dependencia física. ${ }^{12}$

5. Menores trastornos psiquiátricos, comparados con fumadores diarios. ${ }^{5}$

6. Mayores intentos para dejar de fumar. ${ }^{9}$

7. Uso asociado a situaciones sociales (reuniones, en bares, cuando beben, cuando están con otros fumadores).12,13 
8. Angustia afectiva asociada a consumo o recaída. ${ }^{14}$

9. Asociación de ingesta de alcohol con tabaco, mayor deseo de fumar «craving» cuando se ingiere alcohol. ${ }^{15}$

Se ha reportado que los fumadores de ocasión tienen puntajes más bajos en el test de dependencia a la nicotina de Fagerström, en un 98\% se ha encontrado dependencia física leve, en comparación frente a los otros más intensos, quienes expresan una notoria sujeción a dicha droga. ${ }^{16}$ Dicha sumisión a tal alcaloide se puede medir por el número de cigarros fumados por día y el tiempo entre que se despierta y fuma el primer cigarro, la cual es una medida de la dependencia física, porque se relaciona con la intensidad de los síntomas de abstinencia después de la privación nocturna. ${ }^{17}$

El modelo de regulación de nicotina ayuda a explicar el comportamiento de aquellos que fuman diario, ya que la subordinación a esta sustancia se considera como el factor determinante del consumo. Quienes lo hacen con mucha frecuencia durante el día, es porque buscan mantener cierta concentración plasmática del alcaloide y así evitar el síndrome de abstinencia. ${ }^{18}$ Sin embargo, este modelo no explica el comportamiento de aquellos usuarios esporádicos. Por ello, evaluar los niveles de dependencia en este tipo de consumidores del cigarro puede ser complejo. Se ha reportado que quienes son adeptos ocasionales afirman que su comportamiento está motivado por la experiencia de placer durante el uso, en contraste con los motivos por los cuales consumen las personas con mayor adicción (disminución del malestar y la habituación). ${ }^{19}$ Un estudio informó que $75 \%$ de los usuarios de ocasión fuman sólo cuando realmente pudieron disfrutar de la experiencia, en contraste con $17 \%$ de los que lo acostumbran diariamente, que coinciden con tal afirmación. Estos hallazgos sugieren que los fumadores eventuales son movidos por el reforzamiento positivo en la conducta de fumar, en cotejo con los consumidores diarios en quienes su conducta se debe a un reforzador negativo que alivia las manifestaciones de abstinencia, además de exponerse igualmente a reforzadores positivos. ${ }^{20}$ Otra motivación positiva es asociar el consumo del tabaco con las reuniones sociales, haya o no ingesta de alcohol o de otras drogas, conducta frecuente en muchos de ellos.

En un estudio se demostró que esos fumadores ocasionales no incrementan su uso cuando se les cambian los cigarros por unos de muy bajo contenido de nicotina, incluso se observó una disminución; ${ }^{21}$ lo cual soporta la teoría de que el consumo en ese grupo podría no estar dirigido enteramente por el ingesta de tal droga.

Es importante considerar que muchos de esos usuarios incidentales son jóvenes quienes están comenzando la adicción. ${ }^{22}$ Para la sanidad pública es redituable dirigir campañas de cesación a los jóvenes que fuman de vez en cuando, ya que algunos de ellos probablemente escalarán el uso y, eventualmente, se convertirán en fumadores diarios. ${ }^{22,23}$ Valdría la pena generar mayor evidencia científica intentando determinar si es una etapa transicional o puede ser un patrón permanente.

Se ha advertido que, en comparación con esos consumidores reincidentes, los ocasionales muestran una variabilidad significativa en la identificación como fumadores, percibiéndose muchas veces como no fumadores. ${ }^{11}$ Esto podría generar un subregistro en prevalencias, ya que podrían estar respondiendo con negativa a la pregunta de si son fumadores y a su vez estar subestimando sustancialmente los riesgos para la salud relacionados con su patrón de utilización de tabaco.

\section{DAÑOS A LA SALUD ASOCIADOS}

El tabaco es perjudicial en todas sus modalidades y no existe un nivel seguro de exposición al mismo. Se ha identificado que un consumo ocasional se asocia a casi el mismo peligro de enfermedad cardiovascular que el tabaquismo diario en relación con los no fumadores, a un elevado riesgo de mortalidad cardiovascular (RR: 1.5, IC de 95\%: 1.0-2.3), ${ }^{24}$ y con mayor posibilidad de desarrollar cáncer de pulmón. Además, el riesgo de mortalidad por todas las causas en los hombres quienes fuman intermitentemente aumenta significativamente $(\mathrm{OR}=1.6, \mathrm{IC}$ del 95\%: 1.3-2.1). ${ }^{24,25}$

Es necesario señalar que los consumidores ligeros pueden tener daños en su organismo, enfrentan peligros de padecer alteraciones cardíacas debido a presión arterial alta y arterias obstruidas por colesterol, padecer un aneurisma aórtico, muerte prematura por enfermedad cardiovascular, tumores de pulmón, esófago, estómago y páncreas, infecciones del tracto respiratorio, retraso en la concepción en mujeres y peor función de los espermatozoides en los hombres, rehabilitación más tardada de un cartílago rasgado y otras lesiones, cataratas en los adultos mayores y, en general, peor calidad de vida relacionada con la salud. Los peligros van desde un aumento de 50\% en la recuperación de un cartílago desgarrado (más lenta), hasta un aumento de 500\% para el cáncer de pulmón en las mujeres. El tabaquismo ocasional también puede contribuir a la enfermedad pulmonar obstructiva crónica (bronquitis crónica o enfisema), accidente cerebrovascular, enfermedad arterial periférica, cáncer de mama y otras afecciones. ${ }^{26}$

Un metaanálisis sobre desórdenes coronarios y accidente cerebrovascular encontró que el riesgo de sufrir alguno de esos padecimientos incrementa más de $30 \%$ en usuarios quienes sólo consumen un cigarro al día. ${ }^{27}$ En Brasil, una encuesta nacional encontró del mismo modo que en usuarios episódicos y ligeros del cigarrillo se incrementa la 
alarma de padecer enfermedades pulmonares $(\mathrm{OR}=2.2$ y 1.8 , respectivamente). ${ }^{28}$ Actualmente hay líneas de investigación pendientes, las cuales podrían brindar un notable comprensión y caracterización en la población de fumadores ocasionales.

Posibles líneas de investigación pendientes en la población de fumadores ocasionales:

- Definir conducta longitudinal de los fumadores esporádicos. Es una etapa transicional a fumar diario o al abandono del tabaquismo; o puede ser permanente.

- Qué tanto se asocia al uso concomitante de cigarrillos electrónicos, alcohol y otras drogas y a enfermedad mental en contraste con los fumadores diarios.

- Explorar variables físicas, psicológicas y socioeconómicas en los fumadores ocasionales.

- Qué tanto se benefician del uso de medicamentos para dejar de fumar.

- Algoritmos de tratamientos que consideren los diferentes patrones de consumo en tabaco.

- Cómo abordar y tratar a los llamados «fumadores sociales».

\section{PROPUESTA DE INTERVENCIÓN}

Es indispensable mejorar el cribado clínico del tabaquismo intermitente u ocasional, a fin de lograr un registro más preciso que permita brindar atención integral a dicha población. También es preponderante el desarrollo de estrategias y de abordajes terapéuticos procurando sensibilizar y tratar a los fumadores intermitentes, ya que éstos poseen características muy particulares. Las recomendaciones para aproximárseles consisten en: consejo breve a todo paciente que consuma tabaco, aún con un empleo «ligero» u «ocasional»; utilizar los componentes de la entrevista motivacional enfatizando en las consecuencias asociadas al consumo, reforzando el que ellos poseen la ventaja de tener mayor autocontrol en su uso y combinarlo con un plan para renunciar a fumar, así como buscar otros reforzadores positivos (conductas placenteras) las cuales compitan con el consumo del cigarro. Por último, si éstos no se encuentran interesados, el profesional de la salud debe hacer hincapié en que el tabaquismo esporádico aumenta la probabilidad de que se conviertan en consumidores diarios y elevan el riesgo de padecer una enfermedad asociada.

Heffner et al..$^{22}$ encontraron que jóvenes quienes fuman con diversos patrones de consumo mostraban interés similar en tratamientos de cesación y tenían una respuesta similar a la intervención. No se encontraron diferencias entre los grupos de fumadores infrecuentes, ocasionales y asiduos respecto a la receptividad y a la respuesta con una inter- vención motivacional y de entrenamiento en desarrollo de habilidades cognitivo conductuales.

La medicación con vareniclina puede ser una alternativa, ya que se ha demostrado que un tratamiento de doce semanas tiene una efectividad similar en fumadores pesados y en ligeros, mostrando tasas de abstinencia similares a los tres y seis meses de seguimiento. ${ }^{29}$

Un estudio que evaluó las percepciones de los procedimientos para cesar de fumar encontró que no existían diferencias entre esa división de pesados y los ligeros; ambos grupos consideraban que tanto un asesoramiento especializado como un complemento farmacológico puedes serles de utilidad para dejar el cigarro. ${ }^{30}$

Otro estudio evaluó creencias acerca de la abstinencia entre fumadores diarios y ocasionales, encontraron que los intermitentes reportaban menores niveles de moderación, deseos reducidos de inhalar tabaco, mayor autoeficacia al enfrentarse a los estímulos externos de fumar y valoraban menos los efectos del cigarro, en comparación con aquellos fumadores consuetudinarios. Durante la intervención, los usuarios esporádicos generaron escasos beneficios de renunciar a la adicción, pero igualmente enfrentaron menores barreras que quienes fuman obstinadamente. ${ }^{31}$

Existe evidencia de que, al igual que con fumadores diarios, la combinación de psicoterapia y tratamiento farmacológico incrementa la tasa de abstinencia en los ocasionales. ${ }^{32}$

\section{CONCLUSIONES}

Al considerar lo anterior, parece evidente que a tales fumadores ocasionales y ligeros se les debe ofrecer ayuda para la cesación, considerando que, aunque su uso sea más espaciado y el número de cigarros sea menor, están en riesgo de incrementar su utilización gradualmente, sobre todo los jóvenes, y pueden desarrollar enfermedades asociadas al tabaco. Este grupo de fumadores se benefician de las intervenciones convencionales de la misma manera en la que lo hacen los consumidores asiduos. Se pueden aprovechar los bajos niveles de dependencia y la disminuida percepción de barreras para dejar de fumar, buscando guiar a los fumadores de ocasión y ligeros hacia la abstinencia total. No es deseable que los profesionales de la salud minimicen los peligros del consumo bajo o intermitente, sino que se incremente la motivación en dirección al abandono total.

\section{REFERENCIAS}

1. Instituto Nacional de Psiquiatría Ramón de la Fuente Muñiz, Instituto Nacional de Salud Pública, Secretaría de Salud. Encuesta Nacional de Consumo de Drogas, Alcohol y Tabaco 2016-2017: Reporte de Tabaco. México: INPRFM; 2017 [citado el 24 de octubre de 2020]. Disponible en: http://www.gob.mx/salud\%7Cconadic/acciones-y- 
programas/encuesta-nacional-de-consumo-de-drogas-alcohol-ytabaco-encodat-2016-2017-136758

2. Organización Panamericana de la Salud, Instituto Nacional de Salud Pública. Encuesta global de tabaquismo en adultos. México 2015. Cuernavaca, México: INSP/OPS; 2017 [citado el 24 de octubre de 2020]. Disponible en: https://www.who.int/tobacco/surveillance/ survey/gats/mex-report-2015-spanish.pdf

3. Tobacco Product Use and Cessation Indicators Among Adults - United States, 2018 I CDC [Internet]. 2019 [citado el 11 de noviembre de 2020]. Disponible en: https://www.cdc.gov/tobacco/data_statistics/ mmwrs/byyear/2019/mm6845a2/index.html

4. World Health Organization. Tobacco [Internet]. [Citado el 12 de noviembre de 2020]. Disponible en: https://www.who.int/ westernpacific/health-topics/tobacco

5. Reyes-Guzman CM, Pfeiffer RM, Lubin J, Freedman ND, Cleary SD, Levine $\mathrm{PH}$, et al. Determinants of light and intermittent smoking in the U.S.: results from three pooled national health surveys. Cancer Epidemiol Biomarkers Prev. 2017;26(2):228-239. Available in: https:// doi.org/10.1158/1055-9965.epi-16-0028

6. Romero DR, Pulvers K, Scheuermann TS, Ahluwalia JS. Psychosocial and behavioral characteristics among subgroups of nondaily college student smokers. Tob Use Insights. 2014;7:15-19. Available in: https:// doi.org/10.4137/tui.s13440

7. Cabriales JA, Maldonado BS, Cooper TV. Smoking transitions in a sample of Hispanic daily light and intermittent smokers. Addict Behav. 2016;62:42-46. Available in: https://doi.org/10.1016/j. addbeh.2016.06.009

8. Ni K, Wang B, Link AR, Sherman SE. Does smoking intensity predict cessation rates? A study of light-intermittent, light-daily, and heavy smokers enrolled in two telephone-based counseling interventions. Nicotine Tob Res. 2020;22(3):423-430. Available in: https://doi. org/10.1093/ntr/nty257

9. Hayes RB, Borrelli B. Differences between Latino daily light and heavier smokers in smoking attitudes, risk perceptions, and smoking cessation outcome. Nicotine Tob Res. 2013;15(1):103-111. Available in: https://doi.org/10.1093/ntr/nts095

10. Wang TW, Trivers KF, Marynak KL, O'Brien EK, Persoskie A, Liu ST, et al. Harm perceptions of intermittent tobacco product use among U.S. Youth, 2016. J Adolesc Health. 2018;62(6):750-753. Available in: https://doi.org/10.1016/j.jadohealth.2017.12.017

11. Smith DM, O'Connor RJ, Collins RL, Hyland AJ, Kozlowski LT. Correlates of smoker identity among intermittent and light daily young adult smokers: findings from wave 1 of the Population Assessment of Tobacco and Health (PATH) study. Addict Behav. 2019;98:106034. Available in: https://doi.org/10.1016/j. addbeh.2019.106034

12. Shiffman S, Tindle H, Li X, Scholl S, Dunbar M, Mitchell-Miland C. Characteristics and smoking patterns of intermittent smokers. Exp Clin Psychopharmacol. 2012;20(4):264-277. Available in: https://doi. org/10.1037/a0027546

13. Shiffman S, Dunbar MS, Li X, Scholl SM, Tindle HA, Anderson SJ, et al. Smoking patterns and stimulus control in intermittent and daily smokers. PLoS One. 2014;9(3):e89911. Available in: https://doi. org/10.1371/journal.pone.0089911

14. Shiffman S, Scholl SM, Mao J, Ferguson SG, Hedeker D, Tindle HA. Ecological momentary assessment of temptations and lapses in nondaily smokers. Psychopharmacology (Berl). 2020;237(8):2353-2365. Available in: https://doi.org/10.1007/s00213-020-05539-3
15. Peloquin MP, McGrath DS, Telbis D, Barrett SP. Alcohol and tobacco cue effects on craving in non-daily smokers. Exp Clin Psychopharmacol. 2014;22(6):502-510. Available in: https://doi. org/10.1037/a0038250

16. Paz-Ballesteros WC, Zavala-Arciniega L, Gutiérrez-Torres DS, Ponciano-Rodríguez G, Reynales-Shigematsu LM. Evaluación de la dependencia física y psicológica al tabaco en fumadores mexicanos adultos, ENCODAT 2016. Salud Pública Mex. 2019;61(2):136-146. Disponible en: https://doi.org/10.21149/9858

17. Heatherton TF, Kozlowski LT, Frecker RC, Fagerstrom KO. The Fagerstrom test for nicotine dependence: a revision of the Fagerstrom tolerance questionnaire. Br J Addict. 1991;86(9):1119-1127. Available in: https://doi.org/10.1111/j.1360-0443.1991.tb01879.x

18. Prochaska JJ, Benowitz NL. Current advances in research in treatment and recovery: Nicotine addiction. Sci Adv. 2019;5(10):eaay9763. Available in: https://doi.org/10.1126/sciadv. aay 9763

19. Shiffman S, Terhorst L. Intermittent and daily smokers' subjective responses to smoking. Psychopharmacology (Berl). 2017;234(19):29112917. Available in: https://doi.org/10.1007/s00213-017-4682-x

20. Coggins CRE, Murrelle EL, Carchman RA, Heidbreder C. Light and intermittent cigarette smokers: a review (1989-2009). Psychopharmacology (Berl). 2009;207(3):343-363. Available in: https://doi.org/10.1007/s00213-009-1675-4

21. Shiffman S, Mao JM, Kurland BF, Scholl SM. Do non-daily smokers compensate for reduced cigarette consumption when smoking very-low-nicotine-content cigarettes? Psychopharmacology (Berl). 2018;235(12):3435-3441. Available in: https://doi.org/10.1007/s00213018-5056-8

22. Heffner JL, Kealey KA, Marek PM, Bricker JB, Ludman EJ, Peterson AV Jr. Proactive telephone counseling for adolescent smokers: Comparing regular smokers with infrequent and occasional smokers on treatment receptivity, engagement, and outcomes. Drug Alcohol Depend. 2016;165:229-235. Available in: https://doi.org/10.1016/j. drugalcdep.2016.06.014

23. Meshefedjian GA. The smoking spectrum: review of the existing evidence and future directions. J Public Health. 2020;28(1):105-113. Available in: https://doi.org/10.1007/s10389-018-01009-5

24. Luoto R, Uutela A, Puska P. Occasional smoking increases total and cardiovascular mortality among men. Nicotine Tob Res. 2000;2(2):133-139. Available in: https://doi.org/10.1080/713688127

25. Schane RE, Ling PM, Glantz SA. Health effects of light and intermittent smoking: a review. Circulation. 2010;121(13):1518-1522. Available in: https://doi.org/10.1161/circulationaha.109.904235

26. Harvard Health. Light and social smoking carry cardiovascular risks [Internet]. [Citado el 25 de octubre de 2020]. Available in: https:// www.health.harvard.edu/newsletter_article/light-and-social-smokingcarry-cardiovascular-risks

27. Hackshaw A, Morris JK, Boniface S, Tang JL, Milenkovic D. Low cigarette consumption and risk of coronary heart disease and stroke: meta-analysis of 141 cohort studies in 55 study reports. BMJ. 2018;363:j5855. Available in: https://doi.org/10.1136/bmj.k5035

28. Formagini TDB, Gomide HP, Perales J, Colugnati FAB. Prevalence and correlates of light and non-daily smoking in Brazil: results from a nationwide representative survey. Drug Alcohol Depend. 2017;178:15-19. Available in: https://doi.org/10.1016/j. drugalcdep.2017.04.018

29. Ebbert JO, Croghan IT, Hurt RT, Schroeder DR, Hays JT. Varenicline for smoking cessation in light smokers. Nicotine Tob 
Res. 2016;18(10):2031-2035. Available in: https://doi.org/10.1093/ ntr/ntw123

30. Milcarz M, Kaleta D, Bak-Romaniszyn L, Polanska K. Perceived cessation treatment effectiveness among socially disadvantaged light and heavy smokers. Int J Occup Med Environ Health. 2019;32(4):527-536. Available in: https://doi.org/10.13075/ ijomeh.1896.01432

31. Hoeppner BB, Hoeppner SS, Carlon HA, Abry A, Darville A, Rohsenow DJ. Preparing for the quit day: comparing beliefs of nondaily versus daily young adult smokers as they prepare for a quit attempt. Nicotine Tob Res. 2021;23(6):1038-1046. Available in: https:// doi.org/10.1093/ntr/ntaa166

32. Nollen NL, Cox LS, Mayo MS, Ellerbeck EF, Ahluwalia JS. Counseling alone or in combination with nicotine replacement therapy for treatment of black non-daily smokers: a randomized trial. Addiction. 2020;115(8):1547-1560. Available in: https://doi.org/10.1111/add.14948.

Conflicto de intereses: Los autores declaran no tener conflicto de intereses. 